\title{
LUDOPATÍA Y EPILEPSIA EN DEMENCIA FRONTOTEMPORAL: REPORTE DE DOS CASOS
}

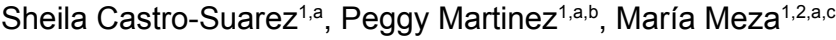

\begin{abstract}
RESUMEN
La demencia frontotemporal es un trastorno neurodegenerativo cuyo subtipo más común es la variante conductual. Es considerada la causa de demencia más frecuente en menores de 60 años. Se presentan dos casos no emparentados cuyos síntomas típicos fueron: deterioro progresivo de la cognición, del comportamiento, alteraciones psiquiátricas como desinhibición, actos impulsivos, apatía, falta de empatía, estereotipias y cambios en los hábitos de alimentación. La resonancia magnética cerebral, en ambos casos, mostró atrofia frontotemporal a predominio izquierdo; en la evaluación neuropsicológica se evidenció alteraciones de las funciones ejecutivas. El primer caso cursó con ludopatía como síntoma inicial, motivo por el cual permaneció por un año en un centro psiquiátrico, siendo el segundo en Latinoamérica y uno de los pocos casos reportados en el mundo. El segundo caso presentó crisis epilépticas en su evolución.
\end{abstract}

Palabras clave: Comportamental; Demencia; Demencia frontotemporal (Fuente: DeCS BIREME)

\section{PATHOLOGICAL GAMBLING AND EPILEPSY IN PATIENTS WITH FRONTOTEMPORAL DEMENTIA: TWO CASE REPORTS}

\begin{abstract}
Frontotemporal dementia is a neurodegenerative disorder of which the behavioral variant is most common. This condition is currently considered the most common cause of dementia in people younger than 60 years. Here, we present two unrelated cases in which the typical symptoms were cognitive and behavioral progressive deterioration and psychiatric disorders such as disinhibition, impulsive acts, apathy, lack of empathy, stereotypies, and changes in eating habits. The first case exhibited pathological gambling as the initial symptom and resided in a psychiatric facility for a year. Notably, this was the second such case in Latin America and one of only a few such cases reported worldwide. The second case presented with epileptic seizures during evolution. In both cases, brain magnetic resonance revealed left-predominant frontotemporal atrophy, and alterations in executive function were evident during neuropsychological assessments.
\end{abstract}

Key words: Behavioral; Dementia; Frontotemporal dementia (Source : MeSH NLM)

\section{INTRODUCCIÓN}

La demencia frontotemporal (DFT) es una enfermedad neurodegenerativa, considerada la segunda causa más común de demencia en personas menores de 60 años de edad; es la demencia con mayor componente genético, resultado de mutaciones en los genes que codifican proteínas que intervienen con el tráfico vesicular, metabolismo de la tau y mutaciones en los genes de las proteínas que contienen valosina y progranulina ${ }^{(1,2)}$. Neuropatológicamente se caracteriza por atrofia, preferentemente, del lóbulo frontal, temporal y la ínsula ${ }^{(1)}$. La frecuencia de DFT es variable y se estimó en un estudio poblacional en nuestro país que reclutó 1532 individuos mayores de 65 años de edad, una prevalencia de DFT de 1,9\% ${ }^{(3)}$. El espectro clínico de la DFT comprende dos grandes subtipos; la variante comportamental y las afasias progresivas primarias que incluye a la afasia progresiva no fluente, la demencia semántica y la afasia logopénica. La demencia frontotemporal variante conductual (DFTc) es la variante más frecuente ya que representa más del $50 \%{ }^{(2)}$.

Las manifestaciones clínicas de la DFTc involucran, inicialmente, cambios conductuales y de la personalidad; un estudio reportó una frecuencia de hasta $92 \%$ de síntomas psiquiátricos en 55 pacientes con DFTc estudiados (4); es por ello que los pacientes con esta variante suelen ser mal diagnosticados como portadores de trastornos psiquiátricos. El espectro clínico completo

\footnotetext{
Instituto Nacional de Ciencias Neurológicas. Lima, Perú.

Facultad de Medicina, Universidad Nacional Mayor de San Marcos. Lima, Perú.

Médica neuróloga; ${ }^{\mathrm{b}}$ magíster en Neurociencias; ${ }^{\mathrm{c}}$ magíster en Neuropsicología
}

Recibido: 20/10/2015 Aprobado: 01/06/2016

Citar como: Castro-Suarez S, Martinez P, Meza M. Ludopatía y epilepsia en demencia frontotemporal: reporte de dos casos. Rev Peru Med Exp Salud Publica. 2016;33(3):588-92. doi: 10.17843/rpmesp.2016.333.2332 
incluye síntomas como apatía, falta de empatía, desinhibición con acciones compulsivas, conductas socialmente embarazosas, alteraciones en sus hábitos alimenticios, como el aumento del apetito y preferencias por los alimentos dulces; además, pueden presentar conductas estereotipadas, las que pueden ir desde rutinas motoras repetitivas hasta obsesiones complejas; estas características clínicas, unidas a los hallazgos de las neuroimágenes y de biomarcadores constituyen los criterios diagnósticos de la DFTc ${ }^{(1,5)}$.

Se presentan dos casos de DFTc no relacionados entre sí, sin antecedentes familiares de demencia, que cursaron con ludopatía uno y epilepsia el otro en la evolución, ambos considerados hallazgos poco frecuentes en esta enfermedad. Los casos cuentan con consentimiento informado, y aprobación del Comité de Ética del Instituto Nacional de Ciencias Neurológicas.

\section{REPORTE DE CASOS}

\section{CASO 1}

Varón de 46 años, monorreno, con 14 años de educación. Trabajó en el área de ventas de una fábrica de algodón. Antecedente de hipertensión arterial y dislipidemia; hermano con diagnóstico de esquizofrenia. Los síntomas se iniciaron a los 39 años de edad caracterizado por jugar incontrolablemente en los casinos; presentaba, además, pensamientos recurrentes y persistentes de limpieza, lo cual afectó de forma negativa su vida personal y familiar; fue hospitalizado en un centro psiquiátrico donde se añadió apatía, desinterés social y agresividad. Seis meses antes de su ingreso al servicio se le observó intranquilo, deseaba constantemente salir de casa, se llenaba la boca excesivamente de comida y comía exageradamente, se comunicaba muy poco, repetía ocasionalmente una sola palabra, presentaba olvidos, dificultad para dormir y vestirse. Al examen neurológico de ingreso mostró una conducta pueril, con risas inapropiadas y jovialidad; orientado en tiempo, espacio y persona; con lenguaje poco fluente, con parafasias y perseveraciones; no repetía frases, pero sí obedecía órdenes simples. Fuerza muscular conservada. Rigidez en miembros inferiores e hiperreflexia generalizada. Presentó reflejo palmomentoneano e imitación.

El resultado de la miniprueba del estado mental fue 26/30 y de la evaluación cognitiva de Montreal fue 16/30. En la evaluación neuropsicológica se evidenció disminución de la atención, dificultades en la planificación, compromiso del lenguaje con disminución de su volumen verbal, alteración de la comprensión y denominación; apraxia, amnesia semántica y episódica. El dominio más alterado fue la función ejecutiva (Tabla 1). La resonancia magnética de encéfalo mostró atrofia cerebral frontotemporal bilateral a predominio izquierdo con ventriculomegalia

Tabla 1. Evaluación neuropsicológica de los casos presentados

\begin{tabular}{|c|c|c|c|}
\hline $\begin{array}{l}\text { Funciones cerebrales } \\
\text { superiores }\end{array}$ & Test utilizado & Caso 1 & Caso 2 \\
\hline Atención y concentración & MMSE & Disminuida & Disminuida \\
\hline $\begin{array}{l}\text { Orientación } \\
\text { temporoespacial }\end{array}$ & MMSE & Conservada & Desorientación \\
\hline $\begin{array}{l}\text { Lenguaje: expresivo, } \\
\text { comprensivo, automático, } \\
\text { denominativo y repetitivo }\end{array}$ & WAIS-III & $\begin{array}{l}\text { Span verbal disminuido, } \\
\text { comprende con dificultad órdenes } \\
\text { complejas, denomina objetos y } \\
\text { repite palabras y frases, menciona } \\
\text { la mayoría de los días de la } \\
\text { semana y meses. }\end{array}$ & $\begin{array}{l}\text { Span verbal muy disminuido, } \\
\text { con ecolalia, obedece órdenes } \\
\text { simples, denominó un solo objeto, } \\
\text { repite solo palabras simples } \\
\text { gramaticalmente, denominó un } \\
\text { solo objeto. }\end{array}$ \\
\hline Gnosia & $\begin{array}{l}\text { Sub test del WAIS-III } \\
\text { Figuras incompletas }\end{array}$ & $\begin{array}{l}\text { Disminución de la discriminación } \\
\text { de detalles }\end{array}$ & $\begin{array}{l}\text { Disminución de la discriminación } \\
\text { de detalles }\end{array}$ \\
\hline Memoria & $\begin{array}{l}\text { WAIS-III } \\
\text { Test de memoria visual } \\
\text { Memorización y } \\
\text { aprendizaje }\end{array}$ & $\begin{array}{l}\text { Memoria inmediata, diferida, } \\
\text { auditiva, icónica, semántica y } \\
\text { episódica disminuida }\end{array}$ & $\begin{array}{l}\text { Memoria inmediata, diferida, } \\
\text { auditiva, icónica, semántica y } \\
\text { episódica disminuida }\end{array}$ \\
\hline Praxia & $\begin{array}{l}\text { Subtest del test de } \\
\text { Barcelona: Praxias }\end{array}$ & $\begin{array}{l}\text { Apraxia constructiva, ideatoria e } \\
\text { ideomotora }\end{array}$ & $\begin{array}{l}\text { Apraxia constructiva, ideatoria e } \\
\text { ideomotora }\end{array}$ \\
\hline $\begin{array}{l}\text { Funciones } \\
\text { ejecutivas }\end{array}$ & $\begin{array}{l}\text { Subtest del NEUROPSI } \\
\text { Subtest de la BANFE-2: } \\
\text { Trail Making test y el } \\
\text { Stroop }\end{array}$ & $\begin{array}{l}\text { No logra hacer la abstracción } \\
\text { de las semejanzas, planificar, ni } \\
\text { seguir las secuencias, es impulsivo } \\
\text { y rompe las reglas. }\end{array}$ & $\begin{array}{l}\text { El paciente no logra hacer la } \\
\text { abstracción de las semejanzas, } \\
\text { intenta seguir la secuencia, pero al } \\
\text { final solo realiza trazos sin objetivo } \\
\text { alguno, no tiene control inhibitorio }\end{array}$ \\
\hline
\end{tabular}




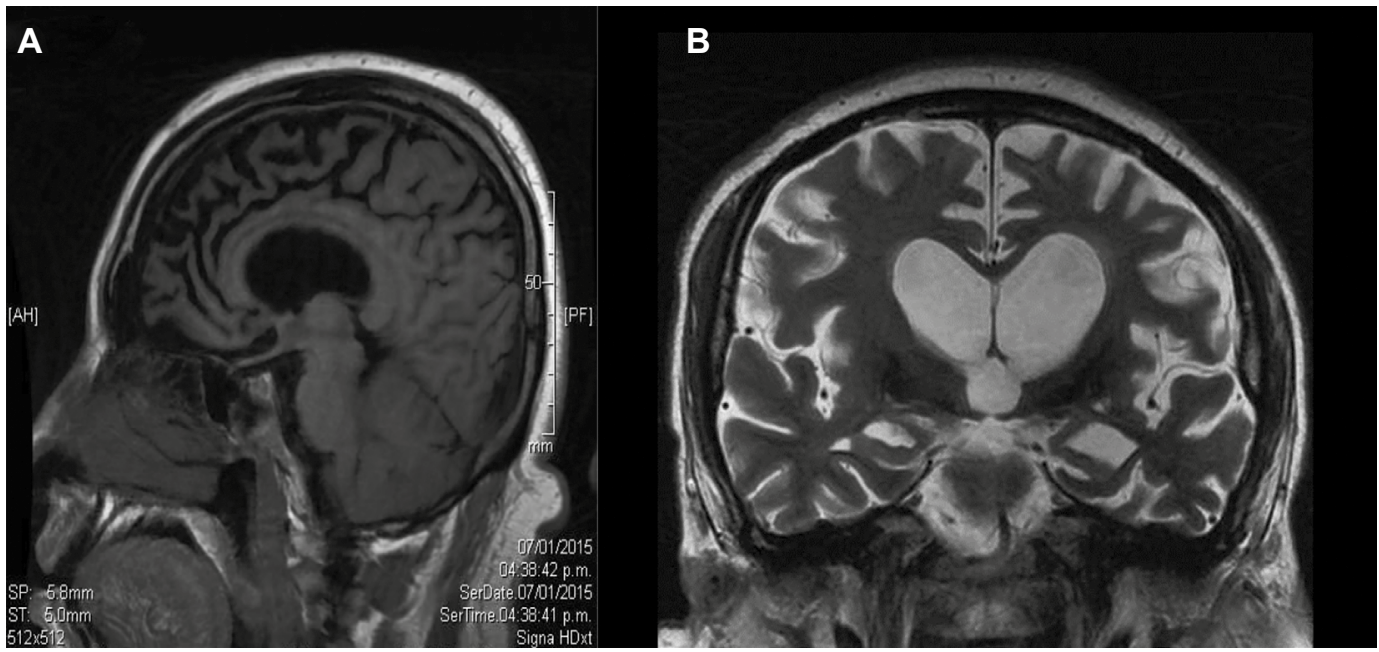

Figura 1. Resonancia magnética cerebral A) protocolo T1 sagital (Caso 1). Se aprecia la atrofia a predominio frontotemporal. B) protocolo T2 coronal (caso 2) atrofia cortical a predominio frontotemporal izquierdo con ventriculomegalia

compensatoria (Figura 1A). Los exámenes hematológicos, bioquímicos, el dosaje de vitamina B12, ácido fólico y hormonas tiroideas fueron normales. Los test serológicos de HIV y RPR fueron negativos.

\section{CASO 2}

Varón de 46 años con instrucción superior, desde hace 5 años presentaba cambios en el comportamiento; saludaba de manera muy efusiva a sus vecinos; a conversar, imitaba los gestos de la otra persona. Un año después se añadieron episodios de conductas inapropiadas, recogía los alimentos que caían al piso con la boca y se guardaba los alimentos en los bolsillos. Notaron también que el paciente pasaba horas lavándose las manos o jugando con el agua en el caño, se mostraba apático y no reconocía a algunos familiares; además, descuidaba su higiene personal, no controlaba esfínteres y presentó disminución progresiva de la producción verbal, ocasionalmente repetía alguna palabra y realizaba movimientos repetitivos de fricción con las manos. La evaluación neurológica al ingreso mostró un paciente temeroso, que llevaba objetos a su boca. Orientado parcialmente en persona, desorientado en tiempo y espacio, la atención y concentración estaban disminuidas, su lenguaje fue poco fluente, ecolálico, no denominaba, no repetía palabras, pero sí obedecía órdenes simples.

La fuerza muscular conservada, presentaba rigidez en extremidades a predominio del lado derecho, hiperreflexia osteotendinosa generalizada, clonus aquileo y Babinski bilateral. Reflejo de prensión y palmomentoneano presentes. Los resultados de la miniprueba del estado mental fue 2/30. La evaluación neuropsicológica evidenció compromiso a predominio de funciones ejecutivas y del lenguaje (Tabla 1). Durante su evolución, el paciente presentó crisis epilépticas parciales con generalización secundaria en más de dos oportunidades. Los hallazgos de la resonancia magnética cerebral fueron compatibles con atrofia cerebral generalizada a predominio frontotemporal (Figura 1B). Los exámenes de laboratorio para descartar demencia secundaria fueron negativos. En el electroencefalograma se halló actividad lenta generalizada que sugiere disfunción cortical difusa. Las crisis epilépticas fueron controladas con medicación antiepiléptica.

\section{DISCUSIÓN}

La DFTc es el subtipo más frecuente de los casos de la DFT, es un síndrome caracterizado por deterioro progresivo de la personalidad, del comportamiento social y la cognición, cuyo diagnóstico se basa en las manifestaciones clínicas (1). La edad promedio de presentación de los casos fue de 40 años, consistente con lo descrito en la literatura, pero se debe tener en cuenta que el rango de edad puede fluctuar entre 31 a 70 años de edad ${ }^{(6,7)}$. La gran mayoría de afectados son menores de 60 años, población económicamente activa, lo cual genera problemas sociales y económicos en la familia ${ }^{(8)}$; en el primer caso reportado, este fue abandonado por su esposa, porque dejó de ser el sustento económico de su familia, en tanto que en el segundo, fue la esposa quien tuvo que asumir los cuidados del paciente y los gastos económicos familiares.

En ambos casos, el síntoma inicial estuvo en relación a desinhibición conductual, en el primer caso manifestado como desorden del impulso (ludopatía), mientras que en 
el segundo predominó la pérdida de las normas sociales; además, mostraron apatía, conducta perseverante, estereotipada o compulsiva; síntomas reportados como los más frecuentes en la presentación de la DFTc (apatía más del $80 \%$, desinhibición $75 \%$ y conducta estereotipada 67\%) ${ }^{(9)}$. Estos síntomas están asociados con disminución de la función de ciertas áreas cerebrales siendo principalmente afectados los circuitos del cíngulo anterior, la ínsula y la corteza órbitofrontal que se encargan de regular el comportamiento ${ }^{(7)}$. El juego patológico como síntoma de la DFTc ha sido reportado en el mundo solo en cinco casos, por lo que el caso que presentamos constituirá el sexto en el mundo y el segundo en Latinoamérica ${ }^{(10)}$. Además, se observó consumo excesivo y no controlado de alimentos y exploración oral de objetos, probablemente como consecuencia de la pérdida de las señales de saciedad; que son típicos de compromiso de la corteza frontoinsular derecha o hipotálamo ${ }^{(7)}$. Los síntomas mencionados en estadios tempranos pueden ser mal interpretados y considerados como una patología psiquiátrica antes de ser catalogados como neurodegenerativos, por ello, permanecen hospitalizados en centros psiquiátricos (11); sin embargo, si el síndrome psiquiátrico es la manifestación inicial y más aun si no se encuentran hallazgos neurológicos en el examen físico, se debe pensar también en una demencia frontotemporal asociada a una expansión del hexanucleótido GGGGCC debida a la mutación en el gen C9Orf72 ${ }^{(12)}$.

Respecto al perfil neuropsicológico, predomina la disfunción ejecutiva, relativa preservación de la memoria episódica y de las habilidades visuoespaciales, como lo hallado en los casos presentados, esta disfunción estaría relacionada a compromiso de estructuras frontosubcorticales (7). Los síntomas descritos en los casos presentados unido a los hallazgos de la resonancia magnética cerebral cumplen criterios para una DFTc probable según lo propuesto por Rascovsky en el año $2011^{(5,9)}$.

En el examen físico neurológico de ambos pacientes se evidenció rigidez, bradicinecia compatible con parkinsonismo, y en la evolución solo el segundo individuo presento crisis epilépticas; este último hallazgo es de presentación rara; sin embargo, los hallazgos mencionados podrían estar en relación a una mutación de la proteína Tau localizada en el cromosoma $17^{(13)}$
Existen reportes en familias portadoras de la mutación P301S con un fenotipo clínico que incluye una demencia frontotemporal de inicio temprano y rápidamente progresivo, parkinsonismo y crisis epilépticas ${ }^{(14,15)}$; por lo cual en presencia parkinsonismo y crisis epilépticas en la evolución de un paciente con probable DFT se plantearía la posibilidad de la presencia de esta mutación genética.

En nuestro país aún no es posible realizar el diagnóstico definitivo de esta entidad debido a que no se cuenta con el material necesario para la tipificación histopatológica de la DFTc (inclusiones de la proteína tau, de la proteína fijadora del ADN TDP-43 y de la proteína fusionada en el sarcoma) ${ }^{(16)}$, ni para la identificación de alguna mutación patogénica, ya que clínicamente podrían ser confundidas con otras entidades como la enfermedad de Alzheimer o con trastornos psiquiátricos ${ }^{(5,17)}$, además la DFTc puede también ser confundida con otras demencias como la degeneración cortico basal, o estar asociada a entidades como la parálisis supranuclear progresiva, donde la correcta aplicación de los criterios diagnósticos cumplirá un rol muy importante; en los casos presentados las características clínicas y las neuroimágenes nos ayudan a identificar con cierto grado de precisión al diagnóstico de DFTc probable, ya que, a pesar de que en ambos casos se halló en el examen físico signos parkinsonianos (rigidez y bradicinecia), no presentan otros hallazgos como distonía, mioclonus, aliens limb, compromiso sensitivo cortical, alteraciones oculomotoras o inestabilidad en la marcha ${ }^{(18)}$.

En conclusión, ante un paciente adulto joven con síntomas psiquiátricos, cambios de la personalidad, en el comportamiento social y el cognitivo, se debe plantear el diagnóstico de demencia frontotemporal antes de pensar en un trastorno psiquiátrico. En estos pacientes las crisis epilépticas no son comunes, pero su presencia podría hacer pensar en un componente genético subyacente en relación a la mutación de la proteína Tau.

Contribuciones de autoría: SC, PM y MM participaron en la concepción, redacción y revisión crítica importante del contenido del manuscrito; así como de su aprobación de la versión final que se publicará.

Fuentes de financiamiento: autofinanciado.

Conflictos de interés: los autores declaran no tener ningún conflicto de interés.

\section{REFERENCIAS BIBLIOGRÁFICAS}

1. Katya R. Variante comportamental de la demencia frontotemporal (DFTc) - Revisión de la Literatura y recomendaciones para el diagnóstico temprano. Acta Neurol Colomb. 2010;26(3,supl.1):29-38.
2. Chow TW,AlobaidyAA.Incorporating new diagnostic schemas, genetics, and proteinopathy into the evaluation of frontotemporal degeneration. Contin (Minneap Minn). 2013;19(2 Dementia):438-56. doi: 10.1212/01.
CON.0000429173.35439.9c.

3. Custodio N, García A, Montesinos R, Escobar J, Bendezú L. Prevalencia de demencia en una población urbana de Lima-Perú: estudio puerta a puerta. An Fac Med. 2008;69(4):233-8. 
4. Krudop WA, Kerssens CJ, Dols A, Prins ND, Möller C, Schouws S, et al. Identifying bvFTD Within the Wide Spectrum of Late Onset Frontal Lobe Syndrome: A Clinical Approach. Am J Geriatr Psychiatry. 2015; 23(10):105666. doi: 10.1016/j.jagp.2015.04.002.

5. Rascovsky K, Grossman M. Clinical diagnostic criteria and classification controversies in frontotemporal lobar degeneration. Int Rev Psychiatry. 2013;25(2):145-58. doi: 10.3109/09540261.2013.763341.

6. Knopman DS, Petersen RC, Edland SD, Cha RH, Rocca WA. The incidence of frontotemporal lobar degeneration in Rochester, Minnesota, 1990 through 1994. Neurology. 2004;62(3):506-8.

7. Miller BL, Dickerson BC, Lucente DE, Larvie M, Frosch MP. Case records of the Massachusetts General Hospital. Case 9-2015. A 31-year-old man with personality changes and progressive neurologic decline. $\mathrm{N}$ Engl J Med. 2015;372(12):1151-62. doi: 10.1056/ NEJMcpc1409839.

8. Dinand C, Nover SU, Holle D, Zischka M, Halek M. What is known about the subjective needs of people with behavioural variant frontotemporal dementia? A scoping review. Health Soc Care Community. 2016;24(4):375-85. doi: 10.1111/hsc.12225.

9. Rascovsky K, Hodges JR, Knopman D, Mendez MF, Kramer JH, Neuhaus J, et al. Sensitivity of revised diagnostic criteria for the behavioural variant of frontotemporal dementia. Brain. 2011;134(Pt 9):245677. doi: 10.1093/brain/awr179.

10. Manes FF, Torralva $T$, Roca $M$, Gleichgerrcht E, Bekinschtein TA, Hodges JR. Frontotemporal dementia presenting as pathological gambling. Nat Rev Neurol. 2010;6(6):347-52. doi: 10.1038/nrneurol.2010.34.

11. Mendez MF, Shapira JS, McMurtray A, LichtE, Miller BL. Accuracy of the clinical evaluation for frontotemporal dementia. Arch Neurol. 2007;64(6):830-5.

12. Carla Gramaglia, Roberto Cantello, Emanuela Terazzi, Miryam Carecchio, Sandra D’Alfonso, Nunzia Chieppa, Francesca Ressico, Maria Cristina Rizza, Patrizia Zeppegno. Early onset frontotemporal dementia with psychiatric presentation due to the C9ORF72 exanucleotide repeat expansion: a case report. BMC Neurol. 2014;14:228. doi: 10.1186/s12883-014-0228-6.

13. Foster NL, Wilhelmsen K, Sima AA, Jones MZ, D'Amato CJ, Gilman S. Frontotemporal dementia and parkinsonism linked to chromosome 17: a consensus conference. Conference Participants. Ann Neurol. 1997;41(6):706-15.

14. Sperfeld AD, Collatz MB, Baier $H$, Palmbach M, Storch A, Schwarz J, et al. FTDP-17: an early-onset phenotype with parkinsonism and epileptic seizures caused by a novel mutation. Ann Neurol. 1999;46(5):708-15.

15. Lossos A, Reches A, Gal A, Newman JP, Soffer D, Gomori JM, et al. Frontotemporal dementia and parkinsonism with the P301S tau gene mutation in a Jewish family. J Neurol. 2003;250(6):733-40.

16. Mackenzie IRA, Neumann M, Bigio EH, Cairns NJ, Alafuzoff I, Kril J, et al. Nomenclature and nosology for neuropathologic subtypes of frontotemporal lobar degeneration: an update. Acta Neuropathol. 2010;119(1):1-4. doi: 10.1007/s00401009-0612-2.

17. Varma AR, Snowden JS, LloydJJ, Talbot PR, Mann DM, Neary D. Evaluation of the NINCDS-ADRDA criteria in the differentiation of Alzheimer's disease and frontotemporal dementia. J Neurol Neurosurg Psychiatry. 1999;66(2):184-8.

18. Williams DR, Litvan I. Parkinsonian syndromes. Continuum (Minneap Minn). 2013;19(5 Movement Disorders):1189-212. doi: 10.1212/01. CON.0000436152.24038.e0.

Correspondencia: Sheila Castro Suarez Dirección: Jr. Ancash 1271 Limal Perú. Teléfono: 01-4117727

Correo electrónico:sheics2@hotmail.com, sheics2@gmail.com

\section{Nuestros artículos se encuentran indizados en:}

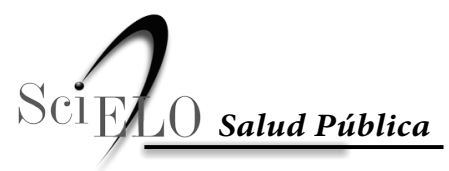

\section{www.scielo.org}

